\title{
LARVICIDAL ACTIVITY OF LANTANA INDICA AND VITEX NEGUNDO ON CULEX QUINQUEFASCIATUS
}

\author{
RATHNASAGAR K, ANAND THIYAGARAJ*
}

Department of Genetic Engineering, Faculty of Engineering and Technology, SRM University, Kattankulathur, Chengalpattu, Tamil Nadu, India. Email: anand.t@ktr.srmuniv.ac.in

Received: 30 January 2018, Revised and Accepted: 30 March 2018

\begin{abstract}
Objectives: The activity of two different leaf extracts of Lantana indica and Vitex negundo is tested against the $3^{\text {rd }}$ and $4^{\text {th }}$ instar Culex quinquefasciatus larvae to evaluate the potency of the extracts as a larvicide and to find an ecologically sustainable alternative to chemical insecticides. A bioinformatics screening approach was performed to evaluate the in vivo results.
\end{abstract}

Methods: The obtained larvae's from nearby water sources were tested with N, N-diethyl-meta-toluamide (DEET) as the positive control which is the commercial chemical mosquito repellent and the solvents used for the respective plant extracts act as the negative control. Petroleum ether (PE), ethyl acetate (EA) and an aqueous (AQ) extract were prepared for both L. indica and V. negundo extracts, and its larvicidal activity was tested. A docking based approach was used to study the inhibitory effect of known active compounds from $L$. indica and $V$. negundo against acetylcholine esterase $(\mathrm{AChE})$ and sterol binding protein as targets.

Results: On comparing the results between three plants extract for its larvicidal activity, the EA extract of $V$. negundo and $L$. indica is found to be potent with a low LC50 value. Further, the docking studies between active compounds of $L$. indica and V. negundo with AChE and Sterol binding protein as targets showed that the compound tangeritin-1 had a good docking score compared to DEET and could be a natural alternative for larvicidal activity in the mosquito.

Conclusion: Individual activity of tangeritin-1 could be further studied with mosquito mortality studies and molecular simulations and develop tangeritin-1 as a potential larvicidal compound for commercial use.

Keywords: Lantana indica, Vitex negundo, N, N-diethyl-meta-toluamide, Culex quinquefasciatus, Molecular docking.

(C) 2018 The Authors. Published by Innovare Academic Sciences Pvt Ltd. This is an open access article under the CC BY license (http://creativecommons. org/licenses/by/4. 0/) DOI: http://dx.doi.org/10.22159/ajpcr.2018.v11i5.25040

\section{INTRODUCTION}

Indian herbs have been long forgotten of its incredible properties and are being exploited by the commercial setups for their various uses and are being produced synthetically. With the advancement in Biotech fields, more and more herbal plants are being studied, and their varied useful properties are being researched extensively. Around 3000 mosquito species belonging to 34 genera in the world have been recorded, of these about 300 transmit Human, animal diseases [1]. The three genera of Mosquitoes are Culex, Anopheles, and Aedes which transmit major mosquito-based vector-borne diseases. Culex quinquefasciatus transmits filariasis caused by the nematode Wuchereria bancrofti. Recent statistics in India showed that 304 million people are exposed to the risk of infection with an estimated 22 million microfilariae carriers and 16 million chronic filariasis case [2]. The epidemic outbreaks of Japanese encephalitis continue to occur year after year, and thousands of young children die of this dreadful disease. Mosquitoes pose the biggest threat acting as a lethal vector spreading dangerous diseases killing millions of people every year. Some of the dangerous diseases caused by mosquitoes are malaria, yellow fever, chikungunya, dengue, and another deadly disease [3]. There are no effective ways to control mosquito population and also they are quite a menace since they bite people and cause an allergic reaction in people. The only effective chemical to control mosquitoes as of now is N, N-Diethyl-meta-toluamide (DEET). DEET has been tested to produce harmful effects in large concentration causing seizures, slurred speech, and coma and in low concentrations, the effects are unknown but certainly will become ecologically magnified [4]. Hence, it is best to search answers from natural sources, not only certain herbs are yielding solutions, but also many common household plants and vegetables serve an answer to our problem [5]. Control of the mosquito larvae is frequently dependent on continued applications of organophosphates and insect growth regulators. An obvious method for the control of mosquito-borne diseases is the use of insecticides, and many synthetic agents have been developed and employed in the field with considerable success. However, one major drawback with the use of chemical insecticide non-selective and could be harmful to other organisms in the environment. It has also provoked undesirable effects, including toxicity to non-target organisms, and fostered environmental and human health concerns. The toxicity problem, together with the growing incidence of insect resistance, has called attention to the need for novel insecticides and more detailed studies of naturally occurring pesticides. Plants are considered as a rich source of bioactive chemicals, and they may be an alternative source of mosquito control agents. Natural products are generally preferred because of their less harmful nature to non-target organisms and due to their innate biodegradability. The current study aims to find some ecologically sustainable alternative for DEET. Vitex negundo Linn and Lantana indica have shown to have been used traditionally in various parts of the world, these plant extract could be used in controlling mosquito breeding and population [6-8]. As larvae eradication is much easier than the elimination of the adult mosquito, new strategies are necessary to control larvae proliferation, like specifically targeting enzymes like AChE [9]. In the current study, larvicidal activity of two plant extracts was studied and the correlated with in silico docking studies of natural compounds of $V$. negundo Linn and $L$. indica against two potential targets of mosquito larvae; acetylcholine esterase (AChE) and sterol carrier protein-2. DEET which is used as positive control has been shown to have an inhibitory effect 
on both the proteins leading to larvicidal effect [10]. The objective was to identify a potential natural compound inhibitor that can replace DEET in larvicidal activity.

\section{METHODS}

Preparation of crude extracts

Petroleum ether extract (PE), ethyl acetate extract (EA), and an aqueous (AQ) extract are the three different extracts prepared from the two plants used in the experiment.

\section{Plant extract preparation}

The leaves of $V$. negundo and L. indica were collected from around Chennai and Coimbatore and were authenticated by botanical experts from Loyola College, Chennai. The leaves were shade dried for 3-4 days till they became brittle and crispy. Then, the leaves were ground into a fine powder. The solvent is added to the leaf powder in an approximate ratio of 3:1 and the setup is left for $48 \mathrm{~h}$ with regular shaking. The extract is then filtered using a filter paper. The extract is subject to rotary vacuum evaporator to get the crude extract. $2 \mathrm{~g}$ of the crude extract is first mixed with $1 \mathrm{~mL}$ Acetone to dissolve and then mixed with $99 \mathrm{~mL}$ of water to make $2 \%$ stock solution. This extract is then tested by releasing them in the larvae system and recording the mortality rate [11].

\section{PE extract preparation}

To $50 \mathrm{~g}$ of the powdered leaf $150 \mathrm{~mL}$ of PE is added and kept in a shaker for a timescale of $48 \mathrm{~h}$ before they were subjected to a rotary vacuum evaporator and a crude extract was formed. EA extract: To $50 \mathrm{~g}$ of the powdered leaf $150 \mathrm{~mL}$ of EA is added and kept in a shaker for a timescale of $48 \mathrm{~h}$ before they were subjected to a rotary vacuum evaporator and a crude extract was formed. Aqueous extract: To $50 \mathrm{~g}$ of the powdered leaf $150-250 \mathrm{~mL}$ of distilled water was added and kept in a shaker for a timescale of $48 \mathrm{~h}$, and the filtered extract is lyophilized, and the extract is prepared [11].

\section{Mosquito larvae collection and breeding}

C. quinquefasciatus eggs (500) have been collected from nearby stagnant water sources such as ponds and lake and verified from entomologist form Entomology Research Centre, Loyola College, Chennai. The eggs once introduced into a pool of dirty water (WHO protocol) undergoes development and starts hatching in a time scale of 2 days. Once the larvae hatch out, they were fed with a mixture of dog biscuit and yeast in the ratio of 3:1 and only a pinch of the mixture are added to a pool of 150 larvae every day to supplement their nutrient needs. At an interval of 1.5 days, the larvae undergo molting and pass four instar stages. The late $3^{\text {rd }}$ instar and the early $4^{\text {th }}$ instar are the best-suited phases for testing the efficacy of the plant extract since they are mature and after which they develop into a pupa [12]. The experiments were performed in a controlled environment.

\section{Extract application procedure}

The $3^{\text {rd }}$ instar and the $4^{\text {th }}$ instar larvae are identified from the tub and isolated separately into a small cup with 10 larvae in a container. EA and PE were the solvents used to prepare the extracts, and an aqueous extract was also prepared. The crude leaf extract of $1 \mathrm{~mL}$ is added to each of the replications in $0.5 \%, 1.0 \%, 1.5 \%$, and $2.0 \%$ strength. The mortality is recorded in $24 \mathrm{~h}$. LC 50 value is calculated for each of the extract being tested.

\section{Docking studies}

Molecular docking was performed using iGEM docking tool a generic docking tool for understanding protein-ligand interaction [12]. This docking protocol has been applied to other targets to find the best docking poses of the ligands. Two proteins AChE and sterol carrier protein and their corresponding PDB are 1UT6 and 2KSH were used as targets and docked with active molecules reported in the literature for $V$. negundo and L. indica which were retrieved in ".mol" format from PubChem database [13]. The strategy was to target the known active compounds against both the active site and allosteric site of the protein [14]. The compounds tangeritin- 1 , beta-caryophyllene, sabinene, betulinic acid, ursolic acid, Vitamin C, and sitosterol were used for docking against two target proteins.

\section{RESULTS}

The mortality rate was recorded at an interval of $24 \mathrm{~h}$ at different concentrations ranging from 0 to $2 \mathrm{mg} / \mathrm{mL}$ of various extracts - $\mathrm{AQ}$, $\mathrm{EA}$, and PE extract of $L$. indica and V. negundo plants (Table 1). The statistical analysis for the mortality was performed and the average

Table 1: LC50 values of aqueous, ethyl acetate, and petroleum ether extract of Lantana indica and V. negundo plants

\begin{tabular}{|c|c|c|c|c|c|c|}
\hline \multirow{2}{*}{$\begin{array}{l}\text { LC50 value and } \\
\text { Standard error } \\
\text { values }\end{array}$} & \multicolumn{2}{|c|}{ Aqueous extract } & \multicolumn{2}{|c|}{ Ethyl acetate extract } & \multicolumn{2}{|c|}{ Petroleum ether extract } \\
\hline & L. indica & V. negundo & L. indica & V. negundo & L. indica & V. negundo \\
\hline LC50 value (mg/mL) & 1.99 & 1.7 & 1.16 & 1.0 & 1.69 & 1.0 \\
\hline Standard error & 0.05 & 0.05 & 0.06 & 0.07 & 0.05 & 0.07 \\
\hline
\end{tabular}

L. indica: Lantana indica, V. negundo: Vitex negundo, L. indica: Lantana indica, V. negundo: Vitex negundo, V. negundo: Vitex negundo

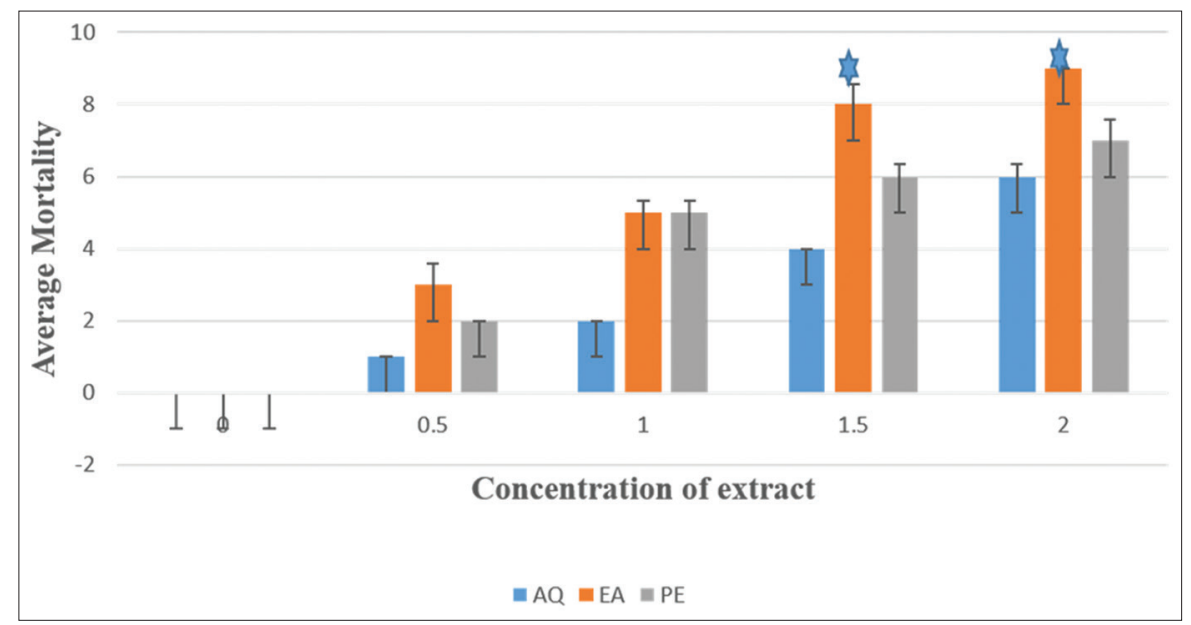

Fig. 1: The average larvae mortality of Culex quinquefasciatus recorded at $24 \mathrm{~h}$ on treatment with aqueous (AQ), EA, and PE extract of Vitex negundo. *Significant concentration of extract with maximum mortality of 10 larvae 
mortality of larvae when exposed to various concentrations for L. indica and $V$. negundo as shown in Figs. 1 and 2.

Among the different plant extracts aqueous extract, EA extract and PE extract of L. indica and $V$. negundo plants, it was observed that higher mortality was seen in PE extract at higher concentrations of plant extract. Further, LC50 calculations were performed. To find LC50 of the plant extracts used in the experiment, substitute $y=5$ in the polynomial equation. On substituting we get $X$ value. Inverse log value of $X$ gives the LC50 value (Table 1 ).

To find standard error approx. S.E. of LC50 $=(\log$ LC84- $\log$ LC16 $) / \sqrt{2}$. The graph was plotted with $\mathrm{X}$-axis as a log of concentration and Y-axis probits of mortality (Fig. 3)

Docking studies were performed between compounds present in L. indica and $V$. negundo and DEET a known inhibitor as control, with two target proteins acetylcholine esterase and sterol carrier protein. Complex with least docking score exhibit high stability. The docking results suggested ligand tangeritin- 1 formed a stable complex with a docking score of -88.8 for acetylcholine esterase (Fig. 4) and -79.5 for sterol carrier protein-2 (Fig. 5), compared to -82.5 for acetylcholine esterase and -78.9 for sterol carrier protein with DEET (Table 2).

\section{DISCUSSION}

The larvicidal effect of $L$. indica and $V$. negundo plant extracts showed increased mortality rate in EA and PE extracts of $V$. negundo compared to $L$. indica other studies for larvicidal activity with different plants also showed increased mortality with PE extracts, suggesting similar

Table 2: A comparative docking score of different active compounds from L. indica and V. negundo against Acetylcholine esterase and sterol carrier protein-2. Ligand tangeretin-1 showing least docking score similar to DEET

\begin{tabular}{lll}
\hline $\begin{array}{l}\text { Active } \\
\text { compounds in } \\
\text { plant extracts. }\end{array}$ & Acetyl choline esterase & $\begin{array}{l}\text { Sterol carrier } \\
\text { protein }\end{array}$ \\
\hline Compounds & Docking energy & Docking energy \\
Tangeretin-1 & -88.8 & -79.5 \\
$\beta$ Caryophyllene & -67.7 & -61.4 \\
Sabinene & -53 & -56.5 \\
Sitosterol & 107.8 & 297.2 \\
Betulinic acid & 441.9 & 556.5 \\
DEET & -82.5 & -78.9 \\
\hline
\end{tabular}

L. indica: Lantana indica, V. negundo: Vitex negundo, DEET: $N$,

$N$-diethyl-meta-toluamide compounds may involve in inducing mortality [15]. The aqueous extract of both plants had lethality only at higher concentration suggesting that compounds are more active in organic solvents due to their chemical composition. Comparing L. indica and V. negundo exhibits more potency in inducing higher mortality at low concentration. Further, the role of individual compounds that contribute to higher mortality was examined. Two targets Acetylcholine esterase and Sterol carrier protein were used as targets for docking studies as they are targeted by many known pesticides like DEET for larvicidal activity. On docking natural compounds of L. indica and V. negundo with PDB structures of AChE and Sterol binding Protein, it was observed that tangeretin- 1 had the least docking score as compared to DEET docking score of -82.5 for acetylcholine esterase. Tangeretin- 1 also exhibited a least docking score of -79.5 compared to -78.9 for DEET with sterol-binding protein. This result suggests that a possible molecular mechanism associated with tangeretin-1 interaction could contribute to the increased mortality rate of the mosquito larvae when treated with $L$. indica and $V$. negundo plant extracts. The amino acids participating in acetylcholine interaction with tangeretin-1 are TYR70, ASP72, TRP84, GLY118, PHE330, TYR334, TRP432, MET436, and HIS440. These amino acids exhibit Van der Waal forces, and no hydrogen bond formation was seen, the nonbonded interactions may potentially induce conformational change and stabilizing the tangeretin-1- AChE complex activity recent Molecular dynamics studies show that Van der Waal forces play a critical role in conformational stability of protein-ligand complex [16]. On comparing the DEET interaction with AChE, it was observed that TRP84 establishes a hydrogen bond with tangeretin-1 and other residues TRP84, PHE330, TRY334, and TRP432 all establish Van der Waal forces, which contributes to the overall stability of the complex [17]. Although the presence of hydrogen in DEET stabilizes the acetylcholine interaction, the higher docking energy of Tangeretin-1 suggest that the Van der Waal forces play a more crucial role in stabilizing the docked complex and can explain the mechanism involved in mortality of mosquito larvae when exposed to crude extracts. A similar docking approach was given to Sterol binding protein-2, where tangeritin-1 showed the least docking energy [18]. The interacting residues are THR35, THR65, ASP88, LYS89, MET90, GLU91, and VAL92 all amino acids contributed to Van der Waal interactions as compared to DEET binding which also had Van der Waal interactions contributing to the stability of the complex. The amino acids participating in DEET interaction are ILE74, ALA81, LYS82, ILE99, PHE100, and GLU103. From the interaction profile, it is evident that the Van der waal forces majorly contribute to docked complex stability in both AChE and Sterol binding protein. Tangeretin-1 establishes Van der waal interaction with a large number of amino acids in both proteins as compared to DEET this possibly establishes the fact that the presence of tangeretin-1 in the crude extract of $L$. indica and $V$. negundo could potentially contribute to increasing larvicidal activity. Tangeretin-1 being an organic substrate with was more effective only in

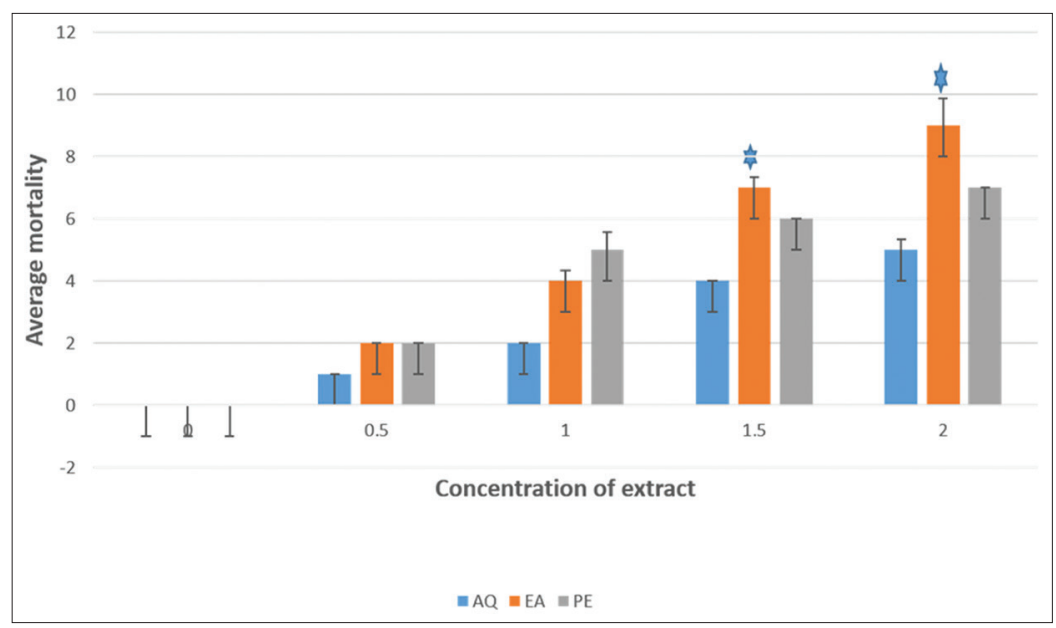

Fig. 2: The average larvae mortality of Culex quinquefasciatus recorded at 24 h on treatment with aqueous (AQ), EA, and PE extract of Lantana indica. *Significant concentration of extract with maximum mortality of 10 larvae 


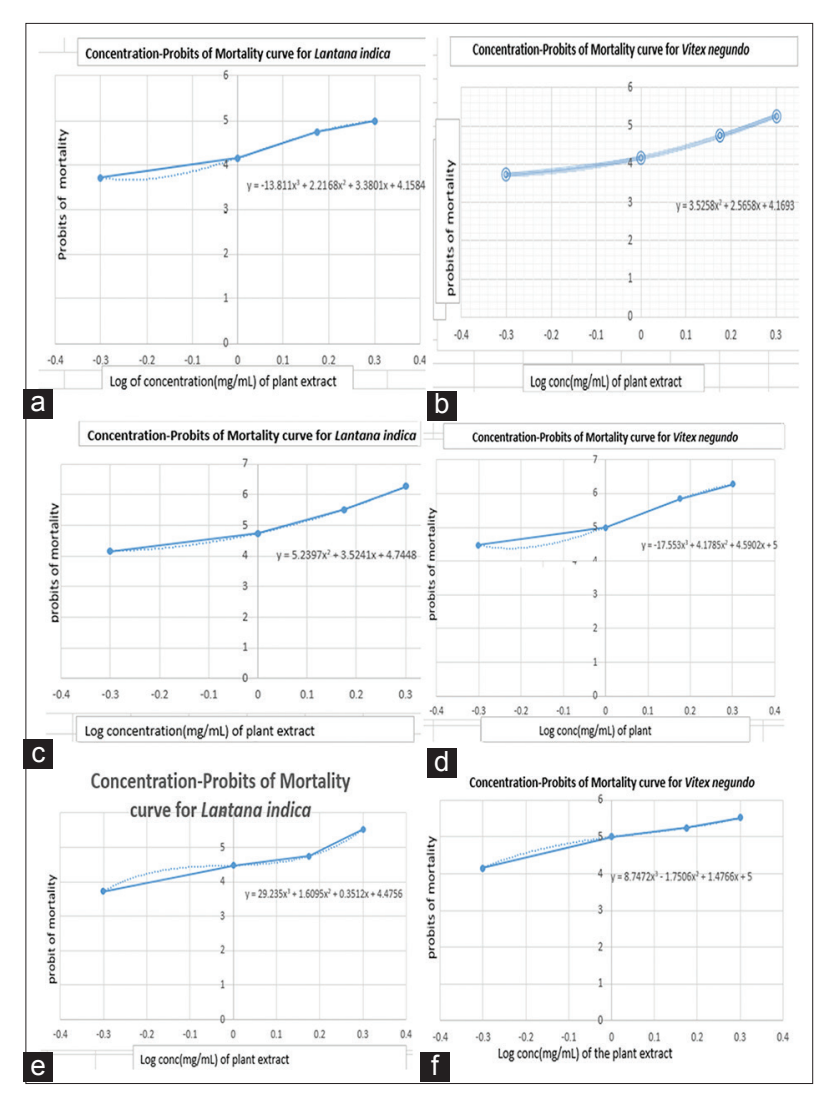

Fig. 3: LC50 calculation for aqueous (AQ), ethyl acetate (EA), and petroleum ether (PE) extracts of Lantana indica and Vitex negundo. X-axis - log of concentration and Y-axis - probits of mortality. (a) LC50 calculation for L. indica AQ extract, (b) LC50 calculation for $V$. negundo AQ extract, (c) LC50 calculation for

$L$. indica EA extract, (d) LC50 calculation for V. negundo EA extract, (e) LC50 calculation for L. indica PE extract, and (f) LC50 calculation for $V$. negundo PE extract

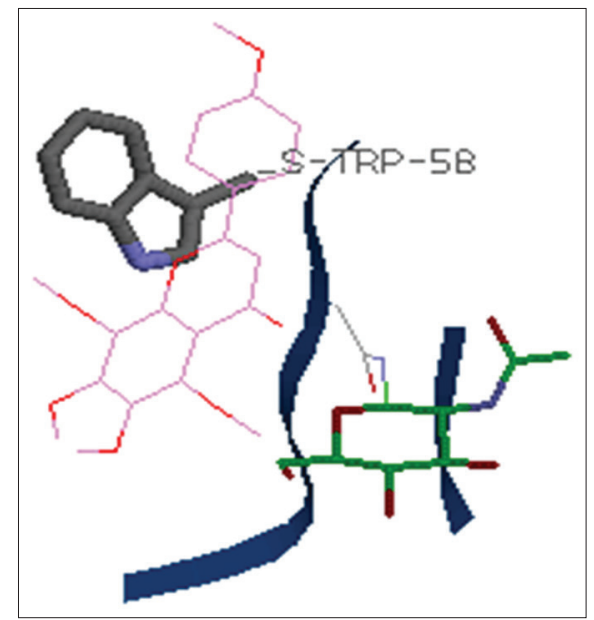

Fig. 4: Docking interaction of acetylcholine esterase with tangeritin-1, at tryptophan 58

$\mathrm{AE}$ and PE extracts where maximum larvicidal activity was seen.

\section{CONCLUSION}

The DEET usage for mosquito larvicidal activity has many harmful effects on prolonged usage. Tangeretin-1, a natural compound shows more strong binding affinity than DEET and does not establish the hydrogen bond as seen in DEET. Hydrogen bond formation in DEET could be a

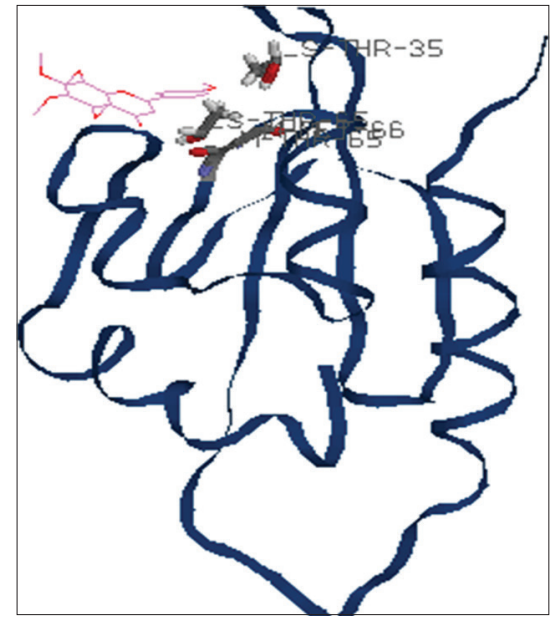

Fig. 5: Docking interaction of sterol carrier protein-2 with tangeritin-1at threonine 35 and threonine 65

possible mechanism of action that contributes to DEET's toxicity. As tangeritin-1 establishes only Van der Waal interaction in protein-ligand stabilization and still exhibit good docking score, they can be used as a potential inhibitor as an alternative to DEET. Commercial usage can be possible with further validation studies such as molecular dynamics and lethal toxicity studies of tangeritin- 1 in future.

\section{CONFLICT OF INTEREST}

No conflict of interest in this study.

\section{REFERENCES}

1. Paul SA. Morphology of tree holes and their mosquitoes collected from Kasmir valley, India. Int J Res Biol Sci 2012;2:94-7.

2. Seufi AM, Galal FH. Role of Culex and Anopheles mosquito species as potential vectors of rift valley fever virus in Sudan outbreak, 2007. BMC Infect Dis 2010;10:65.

3. Gubler DJ. Dengue, urbanization and globalization: The unholy trinity of the 21(st) Century. Trop Med Health 2011;39:3-11.

4. Qiu H, Jun HW, McCall JW. Pharmacokinetics, formulation, and safety of insect repellent N,N-diethyl-3-methylbenzamide (deet): A review. J Am Mosq Control Assoc 1998;14:12-27.

5. Tuetun B, Choochote W, Pongpaibul Y, Junkum A, Kanjanapothi D, Chaithong $\mathrm{U}$, et al. Celery-based topical repellents as a potential natural alternative for personal protection against mosquitoes. Parasitol Res 2008:104:107-15.

6. Haridasan P, Gokuldas M, Ajaykumar AP. Antifeedant effects of Vitex negundo leaf extracts on the stored product pest, Tribolium Castaneum.h (Coleoptera Tenebrionidae). Int J Pharm Pharm Sci 2017;9:17.

7. Hebbalkar DS, Hebbalkar GD, Sharma RN, Joshi VS, Bhat VS. Mosquito repellent activity of oils from Vitex negundo Linn. leaves. Indian J Med Res 1992;95:200-3.

8. Rajashekar Y, Ravindra KV, Bakthavatsalam N. Leaves of Lantana camara Linn. (Verbenaceae) as a potential insecticide for the management of three species of stored grain insect pests. J Food Sci Technol 2014;51:3494-9.

9. Swale DR, Sun B, Tong F, Bloomquist JR. Neurotoxicity and mode of action of N, N-Diethyl-Meta-Toluamide (DEET). PLoS One 2014;9:e103713

10. Dhivya R, Manimegalai K. In silico molecular docking and molecular dynamics applications in the designing of a new mosquito repellent from the plant calotropis gigantea targeting the odorant binding protein of Culex quinquefasciatus. Int J Pharm Phytopharm Res 2013;3:134-8.

11. Sasidharan S, Chen Y, Saravanan D, Sundram KM, Latha LY. Extration, isolationand characterization of bioactive compounds from plan extracts. Afr J Tradit Complement Altern Med 2011;8:1-10.

12. Silva IG, Zanon VO, Silva HH. Larvicidal activity of Copaifera reticulata ducke oil-resin against Culex quinquefasciatus Say (Diptera: Culicidae). Neotrop Entomol 2003;32:729-32.

13. Kim S, Thiessen PA, Bolton EE, Chen J, Fu G, Gindulyte A, et al. Pubchem substance and compound databases. Nucleic Acids Res 2016;44:D1202-13. 
14. Thiyagaraj A, Sunny JS, Hopper W. Effect of toll-like receptor inhibitor imiquimod on IL1R1 interaction with IL1Ra and its SNP variant an in silico approach. Int J Pharm Pharm Sci 2016;8:109-12.

15. Rahuman AA, Gopalakrishnan G, Venkatesan P, Geetha K. Larvicidal activity of some Euphorbiaceae plant extracts against Aedes aegypti and Culex quinquefasciatus (Diptera: Culicidae). Parasitol Res 2008;102:867-73.

16. Gomes DE, Lins RD, Pascutti PG, Lei C, Soares TA. The role of nonbonded interactions in the conformational dynamics of organophosphorous hydrolase adsorbed onto functionalized mesoporous silica surfaces. J Phys Chem B 2010;114:531-40.

17. Carreño Otero AL, Vargas Méndez LY, Duque LJ, Kouznetsov VV. Design, synthesis, acetylcholinesterase inhibition and larvicidal activity of girgensohnine analogs on Aedes aegypti, vector of dengue fever Eur J Med Chem 2014;78:392-400.

18. Li NC, Fan J, Papadopoulos V. Sterol carrier protein-2, a nonspecific lipid-transfer protein, in intracellular cholesterol trafficking in testicular leydig cells. PLoS One 2016;11:e0149728. 